Pacific Journal of Mathematics

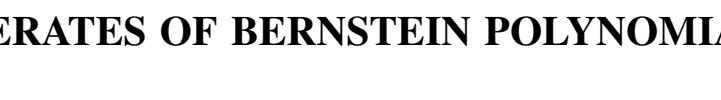




\title{
ITERATES OF BERNSTEIN POLYNOMIALS
}

\author{
R. P. KeLisky AND T. J. RivLIN
}

$B_{n}(f)$ transforms each function defined on $[0,1]$ into its Bernstein polynomial of degree $n$. In this paper we study the convergence of the iterates $B_{n}^{(k)}(f)$ as $k \rightarrow \infty$ both in the case that $k$ is independent of $n$ and (for polynomial $f$ ) when $k$ is a function of $n$.

To each $f(x)$ defined on $I: 0 \leqq x \leqq 1$ there is associated its Bernstein polynomial of degree $n$ defined by

$$
B_{n}(f ; x)=\sum_{k=0}^{n} f\left(\frac{k}{n}\right)\left(\begin{array}{l}
n \\
k
\end{array}\right) x^{k}(1-x)^{n-k} .
$$

It is well known that if $f$ is continuous on $I$, then

$$
\lim _{n \rightarrow \infty} B_{n}(f ; x)=f(x)
$$

uniformly on $I$. (Cf., Lorentz [2] for this and other properties of the Bernstein polynomials used here.) Let $B_{n}(f)$ denote the (polynomial) function defined by (1.1), then for $k>1, B_{n}^{(k)}(f ; x)=B_{n}\left(B_{n}^{\langle k-1)}(f) ; x\right)$ defines, by mathematical induction, a sequence of iterates of the Bernstein polynomials. Our purpose is to study the convergence behavior of this sequence as $k \rightarrow \infty$, both in the case that $k$ is independent of $n$ and when it is a nonconstant function of $n$.

We show in $\S 2$ that $B_{n}^{(k)}(f ; x)$ converges (uniformly) for fixed $n$, to the line segment joining $(0, f(0))$ to $(1, f(1))$, and in $\S 3$ that the sequence $B_{n}^{(g(n))}\left(x^{s} ; x\right)$ with appropriate assumptions on $g(n)$, also converges, for each $s=0,1,2, \cdots$ to a polynomial of degree $s$ whose coefficients we determine explicitly. Finally, in $\S 4$ arbitrary iterates are defined as a natural generalization of the positive integral iterates. When (1.1) is rewritten in conventional polynomial form, it becomes

$$
\begin{aligned}
B_{n}(f ; x) & =\sum_{q=0}^{n}\left\{\left(\begin{array}{l}
n \\
q
\end{array}\right) \sum_{k=0}^{q} f\left(\frac{k}{n}\right)\left(\begin{array}{l}
q \\
k
\end{array}\right)(-1)^{q-k}\right\} x^{q} \\
& =\sum_{q=0}^{n} \Delta_{1 / n}^{q} f(0)\left(\begin{array}{l}
n \\
q
\end{array}\right) x^{q}
\end{aligned}
$$

which reveals that if $f$ is a polynomial of degree $m$, then $B_{n}(f)$ is a polynomial whose degree is at most $\min (m, n)$. Let $s$ be a fixed positive integer satisfying $s \leqq n$. (There is no loss of generality in this restriction on $s$ for $k>1$, since for $s>n, B_{n}^{(k)}\left(x^{s}\right)=B_{n}^{(k-1)}\left(B_{n}\left(x^{s}\right)\right)$ and $B_{n}\left(x^{s}\right)$ is of degree at most $n$.) We consider $f(x)=x^{j}, j=1, \cdots, s$. (1.3) implies that 


$$
\begin{aligned}
B_{n}\left(x^{j}\right)=a_{1 j} x+a_{2} x^{2}+\cdots+a_{j j} x^{j}=\sum_{q=1}^{j} \pi_{q} \sigma_{j}^{q} \frac{1}{n^{j-q}} & x^{q}, \\
& j=1, \cdots, s,
\end{aligned}
$$

where $\sigma_{j}^{q}$ are the Stirling numbers of the second kind (Cf., Jordan [1, pp. 168-173]) defined by

$$
\sigma_{j}^{q}=\frac{(-1)^{q}}{q !} \sum_{k=1}^{q} k^{j}\left(\begin{array}{l}
q \\
k
\end{array}\right)(-1)^{k},
$$

and

$$
\left\{\begin{array}{l}
\pi_{q}=\left(1-\frac{1}{n}\right)\left(1-\frac{2}{n}\right) \cdots\left(1-\frac{q-1}{n}\right), \quad q=2, \cdots, s \\
\pi_{1}=1
\end{array}\right.
$$

2. Limit of the iterates. The study of the iterates of $B_{n}(f ; x)$ for $f(x)=x^{s}$ is considerably simplified if we use the language of linear algebra. There is no loss of generality in this choice of $f(x)$ since $B_{n}$ replaces $f$ by a polynomial.

Let $A$ denote the $s \times s$ upper triangular matrix whose entries $a_{i j}$ are defined in (1.4), i.e.,

$$
a_{i j}= \begin{cases}\pi_{i} \sigma_{j}^{i} n^{i-j}, & i \leqq j \\ 0 & i>j\end{cases}
$$

Let $e_{s}$ be the column vector of $s$ components, the first $s-1$ components being zero and the last one. Then

Lemma 1. If $A^{k} e_{s}=\left(\alpha_{1}^{(k)}, \cdots, \alpha_{s}^{(k)}\right)^{T}$, then

$$
B_{n}^{(k)}\left(x^{s}\right)=\alpha_{1}^{(k)} x+\alpha_{2}^{(k)} x^{2}+\cdots+\alpha_{s}^{(k)} x^{s}, \quad k=1,2, \cdots .
$$

Proof. If $p(x)=c_{1} x+c_{2} x^{2}+\cdots+c_{s} x^{s}$ (for example, $p(x)=$ $\left.B_{n}^{(j)}\left(x^{s}\right)\right)$ and

$$
\begin{aligned}
B_{n}(p)=d_{1} x+d_{2} x^{2}+\cdots+d_{s} x^{s} & =\sum_{j=1}^{s} c_{j}\left(a_{1 j} x+\cdots+a_{s j} x^{s}\right) \\
& =\sum_{l=1}^{s} \sum_{j=1}^{s} c_{j} a_{l j} x^{l},
\end{aligned}
$$

then $\left(d_{1}, \cdots, d_{s}\right)^{T}=A\left(c_{1}, \cdots, c_{s}\right)^{T}$. The lemma now follows by mathmatical induction on $k$.

Lemma 2. The eigenvalues of $A$ are $\pi_{1}, \pi_{2}, \cdots, \pi_{s}$.

$$
\text { Proof. } a_{i i}=\pi_{i}, i=1, \cdots, s \text {, and } a_{i j}=0 \text { if } i>j \text {. }
$$


Let $\Lambda$ denote the $s \times s$ matrix with the eigenvalues of $A, \pi_{1}, \cdots, \pi_{s}$ on the main diagonal and zeros everywhere else. Let $V$ denote the matrix of eigenvectors of $A$, normalized so that the entries on its main diagonal are all $1 . V$ is upper triangular and its entries are, in general, functions of $n$. Since $A V=V A$ we conclude that

$$
A^{k}=V \Lambda^{k} V^{-1} .
$$

Essentially, the following arguments rest on the observation that $A^{k}$ is known to us and $V$ and its inverse are independent of $k$.

Lemma 3. If $V^{-1}=\left(\bar{v}_{i j}\right)$ then $\bar{v}_{1 j}=1, j=1, \cdots, s$.

Proof. Let $U$ be the eigenmatrix of $A^{T}$, i.e.,

$$
A^{T} U=U A \text {. }
$$

Let $U$ (which is lower triangular) be normalized so that the entries on its main diagonal are all 1 . Since $B_{n}\left(x^{j} ; 1\right)=1$ the column sums of $A$ are all 1 and hence the row sums of $A^{T}$ are all 1 . The first column of $U$ is the eigenvector associated with the eigenvalue $\pi_{1}=1$, and hence consists of all entries 1 . Due to the way we have normalized $V$ and $U$ we know that $U^{r}=V^{-1}$ and the lemma is proved.

LEMMA 4. If $n$ is fixed

$$
\lim _{k \rightarrow \infty} A^{k} e_{s}=(1,0,0, \cdots, 0)^{T} .
$$

Proof. The entries on the main diagonal of $\Lambda^{k}$ are $\pi_{1}^{k}, \cdots, \pi_{s}^{k}$ and

$$
\begin{aligned}
& \lim _{k \rightarrow \infty} \pi_{j}^{k}=0, \quad j=2, \cdots, s \\
& \lim _{k \rightarrow \infty} \pi_{1}^{k}=1 .
\end{aligned}
$$

Thus, as $k \rightarrow \infty, V \Lambda^{k} V^{-1}$ approaches a matrix whose first row consists of all 1 's, by Lemma 3, and the rest of whose elements are all 0 . Clearly,

$$
(1,0,0, \cdots, 0)^{r}=\left(\lim _{k \rightarrow \infty} A^{k}\right) e_{s}=\lim _{k \rightarrow \infty}\left(A^{k} e_{s}\right) .
$$

THEOREM 1. If $n$ is fixed then

$$
\lim _{j \rightarrow \infty} B_{n}^{(j)}(f ; x)=f(0)+(f(1)-f(0)) x, \quad 0 \leqq x \leqq 1 .
$$

Proof. Let $B_{n}(f ; x)=\alpha_{0}+\alpha_{1} x+\cdots+\alpha_{n} x^{n}$, then $B_{n}^{(j)}(f ; x)=\alpha_{0}+\alpha_{1} B_{n}^{(j-1)}(x ; x)+\alpha_{2} B_{n}^{(j-1)}\left(x^{2} ; x\right)+\cdots+\alpha_{n} B_{n}^{(j-1)}\left(x^{n} ; x\right)$; 
hence, in view of Lemma 1 and Lemma 4 , with $s=1,2, \cdots, n$,

$$
\begin{aligned}
\lim _{j \rightarrow \infty} B_{n}^{(j)}(f ; x) & =\alpha_{0}+\left(\alpha_{1}+\cdots+\alpha_{n}\right) x \\
& =f(0)+(f(1)-f(0)) x .
\end{aligned}
$$

REMARK. The convergence in (2.4) is uniform since we have a sequence of polynomials of fixed degree approaching a fixed polynomial of the same degree for all $x$ on a bounded interval. Also we have used the obvious fact that $B_{n}(1)=1$, all $n$.

It is a curious fact that the matrix $V$ has the property that $v_{i j}$ is independent of $n$, for $j=1,2,3$. We have, when $s=3$,

$$
V=\left(\begin{array}{rrc}
1 & -1 & 1 / 2 \\
0 & 1 & -3 / 2 \\
0 & 0 & 1
\end{array}\right)
$$

Let $p_{2}(x)=-x+x^{2}$ and $p_{3}(x)=(1 / 2) x-(3 / 2) x^{2}+x^{3}$, then we conclude that,

$$
\begin{aligned}
& B_{n}^{(j)}\left(p_{2}\right)=\left(1-\frac{1}{n}\right)^{j} p_{2}, \quad j=0,1,2, \cdots \\
& B_{n}^{(j)}\left(p_{3}\right)=\left[\left(1-\frac{1}{n}\right)\left(1-\frac{2}{n}\right)\right]^{j} p_{3} .
\end{aligned}
$$

These results should be contrasted to the well-known remark (Cf., Schoenberg [3]) that the Bernstein operators are "poor reproducers", in that they never reproduce polynomials of degree greater than 1 .

3. Limit of the coupled iterates. Suppose $f(x)=x^{s}$. Theorem 1 tells us that for fixed $n, B_{n}^{(j)}\left(x^{s}\right) \rightarrow x$ as $j \rightarrow \infty$, while according to (1.2), $B_{n}\left(x^{s}\right) \rightarrow x^{s}$ as $n \rightarrow \infty$. Thus, it is of interest to "play-off" the upper and lower subscripts in $B_{n}^{(j)}\left(x^{s}\right)$, by considering $j=g(n)$. To this end we must examine the behavior of the eigenmatrix, $V$, as $n \rightarrow \infty$.

Let the elements of $V$ be $v_{i j}\left(=v_{i j}(n)\right)$. For $j=1, \cdots, s$ we have

$$
A\left(v_{1 j}, \cdots, v_{s j}\right)^{T}=\pi_{j}\left(v_{1 j}, \cdots, v_{s j}\right)^{T} .
$$

We examine these linear equations more closely. Since $V$ is upper triangular,

$$
v_{i j}=0, \quad i=j+1, \cdots, s,
$$

and because of the way we have normalized $V$

$$
v_{j j}=1 \text {. }
$$

It remains, then, to determine the behavior of $v_{i j}(n), i<j$, as $n \rightarrow \infty$. 
We consider the relevant linear equations from (3.1) (and write $v_{i}$ in place of $v_{i j}$ for simplicity)

$$
\begin{aligned}
a_{j-1, j-1} v_{j-1}+a_{j-1}, j & =\pi_{j} v_{j-1} \\
a_{j-2, j-2} v_{j-2}+a_{j-2, j-1} v_{j-1}+a_{j-2, j} & =\pi_{j} v_{j-2} \\
\vdots & \\
a_{11} v_{1}+a_{12} v_{2}+\cdots+a_{1, j-1} v_{j-1}+a_{1, j} & =\pi_{j} v_{1} .
\end{aligned}
$$

Define $\pi_{i j}=\pi_{i}-\pi_{j}$, let $P$ denote the determinant $\left|p_{i j}\right|$ such that

$$
p_{i j}= \begin{cases}a_{i j} & i<j \\ \pi_{i j} & i=j \\ 0 & i>j\end{cases}
$$

then

$$
P=\prod_{k=1}^{j-1} \pi_{k j}
$$

Let $P^{(i)}$ denote the determinant identical to $P$ except that the $i$-th column of $P$ is replaced by $\left(-a_{1 j},-a_{2 j}, \cdots,-a_{j-1, j}\right)$. Then, if we solve (3.4) for $v_{i}\left(=v_{i, j}\right)$ by Cramer's rule, we obtain

$$
v_{i}=\frac{P^{(i)}}{P} \text {. }
$$

If we denote by $P_{p j}^{(i)}$ the minor of $-a_{p j}$ in $P^{(i)}$, then $P_{p j}^{(i)}$ is upper triangular and

$$
(-1)^{i+p} P_{p j}^{(i)}= \begin{cases}0 & p<i \\ P / \pi_{i j} & p=i \\ a_{i, i+1} a_{i+1, i+2} \cdots a_{p-1, p} P / \prod_{k=i}^{p} \pi_{k j} & p>i\end{cases}
$$

Now,

$$
(-1)^{i+p+1} a_{p j} P_{p j}^{(i)} / P= \begin{cases}-a_{i j} / \pi_{i j} & p=i \\ \frac{(-)^{i+p+1} a_{p j} a_{i, i+1} \cdots a_{p-1, p}}{\prod_{k=i}^{p} \pi_{k j}} & p>i\end{cases}
$$

and for $q<j$,

$$
\begin{aligned}
\pi_{q j} & =\pi_{q}\left[1-(1-q / n) \cdots\left(1-\frac{j-1}{n}\right)\right] \\
& =\pi_{q}\left\{\frac{1}{n}[q+(q+1)+\cdots+(j-1)]+O\left(n^{-2}\right)\right\}
\end{aligned}
$$


as $n \rightarrow \infty$. Since $\pi_{i} \rightarrow 1$ as $n \rightarrow \infty$, we obtain, in view of (3.6), (3.7), and $(2.1)$,

$$
\lim _{n \rightarrow \infty} \frac{a_{p j} P_{p j}^{(i)}}{P}=0, \quad p<j-1
$$

while

$$
\lim _{n \rightarrow \infty} \frac{a_{j-1, j} P_{j-1, j}^{(i)}}{P}=\left\{\prod_{t=i}^{j-1}\left(\frac{j-t}{2}\right)(j+t-1)\right\}^{-1} \sigma_{t+1}^{t}
$$

Thus, we obtain, finally, that

(3.8) $\lim _{n \rightarrow \infty} v_{i j}=v_{i j}^{*}=(-1)^{j+i} 2^{j-i} \frac{\prod_{t=i}^{j-1}\left(\begin{array}{c}t+1 \\ 2\end{array}\right)}{[(j-i) !]^{2}\left(\begin{array}{c}2 j-2 \\ j-1\end{array}\right)}, i=1, \cdots, j-1$.

where we have used the fact that (Cf., Jordan [1])

$$
\sigma_{t+1}^{t}=\left(\begin{array}{c}
t+1 \\
2
\end{array}\right)
$$

(3.2), (3.3), and (3.8) give the limit of $V$ as $n \rightarrow \infty$. In an entirely analogous fashion, with $A^{T}$ in place of $A$, we may obtain the limit of $V^{-1}$ as $n \rightarrow \infty$. We suppress the details, but the result is

$$
\lim _{n \rightarrow \infty} \bar{v}_{i j}=\bar{v}_{i j}^{*}= \begin{cases}0, & i>j \\
1, & i=j \\
2^{j-i} \frac{\prod_{t=i}^{j-1}\left(\begin{array}{c}
t+1 \\
2
\end{array}\right)}{[(j-i) !]^{2}\left(\begin{array}{c}
i+j-1 \\
j-i
\end{array}\right)}, & i<j .\end{cases}
$$

Let us put

$$
E_{j}=\exp \left[-\left(\begin{array}{l}
j \\
2
\end{array}\right)\right]=\lim _{n \rightarrow \infty} \pi_{j}^{n}
$$

THEOREM 2. Suppose $g(n)$ is a nonnegative integer for each $n$, and

$$
\lim _{n \rightarrow \infty} \frac{g(n)}{n}=\alpha
$$




$$
\lim _{n \rightarrow \infty} B_{n}^{(g(n))}\left(x^{s}\right)=\sum_{i=1}^{s} b_{i} x^{i}
$$

where

$$
b_{i}=\frac{i}{s}\left(\begin{array}{l}
s \\
i
\end{array}\right)^{2} \sum_{j=i}^{s} \frac{(-1)^{j+i}\left(\begin{array}{c}
s-i \\
j-i
\end{array}\right)^{2}}{\left(\begin{array}{c}
2 j-2 \\
j-i
\end{array}\right)\left(\begin{array}{c}
j+s-1 \\
s-j
\end{array}\right)} E_{j}^{\alpha}
$$

$i=1, \cdots, s$ (where, when $\alpha=\infty$ in (3.11), we have $E_{1}^{\alpha}=1$ and $E_{j}^{\alpha}=0, j>1$ in $\left.(3.13)\right)$.

Proof. $A^{g(n)}=V \Lambda^{g(n)} V^{-1}$. Now

$$
\lim _{n \rightarrow \infty} \Lambda^{g(n)}=\Lambda^{*}
$$

where $\Lambda^{*}$ is a diagonal matrix with entries $E_{j}^{\alpha}, j=1, \cdots, s$ on its main diagonal.

Let

$$
\lim _{n \rightarrow \infty} V=V^{*}
$$

and

$$
\lim _{n \rightarrow \infty} V^{-1}=\left(V^{-1}\right)^{*}=\left(V^{*}\right)^{-1} .
$$

The entries in $V^{*}$ and $\left(V^{*}\right)^{-1}$ are given by (3.2), (3.3), (3.8), and (3.9). Thus, we may conclude that

$$
V^{*} \Lambda^{*}\left(V^{*}\right)^{-1} e_{s}=\left(\lim _{n \rightarrow \infty} A^{g(n)}\right) e_{s}=\lim _{n \rightarrow \infty}\left(A^{g(n)} e_{s}\right)
$$

and the existence of the limit in (3.12) is established. In order to verify (3.13), we need only note that

$$
\left(b_{1}, \cdots, b_{s}\right)^{T}=V^{*} \Lambda^{*}\left(V^{*}\right)^{-1} e_{s},
$$

so that

$$
b_{i}=\sum_{j=1}^{s} v_{i j}^{*} \bar{v}_{j s}^{*} E_{j}^{\alpha}, \quad i=1, \cdots, s .
$$

REMARK. If $\alpha=0$, then $\Lambda^{*}=I$ and we conclude from (3.14) that $\left(b_{1}, \cdots, b_{s}\right)^{T}=e_{s}$, or $b_{j}=0, j=1, \cdots, s-1, b_{s}=1$. In particular, then, if $g(n) \equiv 0$, we have proved (1.2) for the case $f(x)=x^{s}$. As a curiosity we also note that we have established the seemingly nontrivial identities 


$$
\sum_{j=i}^{s} \frac{(-1)^{j+i}\left(\begin{array}{c}
s-i \\
j-i
\end{array}\right)^{2}}{\left(\begin{array}{c}
2 j-2 \\
j-i
\end{array}\right)\left(\begin{array}{c}
j+s-1 \\
s-j
\end{array}\right)}=0, \quad i=1, \cdots, s-1 .
$$

With some simplification (3.16) may be written in the equivalent form (3.17) which holds for odd $t$ and $n$ positive

$$
\sum_{k=0}^{n}(-1)^{k}\left(\begin{array}{c}
t+k \\
t
\end{array}\right)\left(\begin{array}{c}
2 n+t \\
n-k
\end{array}\right) \frac{2 k+t}{k+t}=0 .
$$

Additionally, since

$$
\sum_{i=1}^{s} a_{i j}=1, \quad j=1, \cdots, s
$$

and

$$
\sum_{j=1}^{s} a_{i j} v_{j k}=\pi_{k} v_{i k}, \quad i=1, \cdots, s ; k=1, \cdots, s,
$$

we obtain, after summing on $i$ on both sides of (3.18) and interchanging the order of summation on the left

$$
\sum_{j=1}^{s} v_{j k}=\pi_{k} \sum_{i=1}^{s} v_{i k},
$$

from which we conclude that, if $\delta_{1 k}$ is a Kronecker delta.

$$
\sum_{i=1}^{s} v_{i k}=\delta_{1 k}
$$

and hence also

$$
\sum_{i=1}^{s} v_{i k}^{*}=\delta_{1 k}
$$

We thus have the seemingly nontrivial identities:

$$
1+\sum_{i=1}^{j-1}(-1)^{j+i} 2^{j-i} \frac{\prod_{t=i}^{j-1}\left(\begin{array}{c}
t+1 \\
2
\end{array}\right)}{[(j-i) !]^{2}\left(\begin{array}{c}
2 j-2 \\
j-i
\end{array}\right)}=0, \quad j=2, \cdots, s,
$$

or, equivalently, if $n \geqq 1$,

$$
\sum_{k=0}^{n}(-1)^{k}\left(\begin{array}{c}
n+k \\
k
\end{array}\right)\left(\begin{array}{l}
n \\
k
\end{array}\right) \frac{1}{k+1}=0 .
$$

4. Iterates of all orders. If $t$ is any real number, $-\infty<t<\infty$, we are now in a position to define $B_{n}^{(t)}(f)$, in a manner consistent 
with our definition when $t$ is a nonnegative integer. We define (4.1) $\quad B_{n}^{(t)}\left(x^{k}\right)=b_{1}(t) x+b_{2}(t) x^{2}+\cdots+b_{k}(t) x^{k}, \quad k=1,2, \cdots$, where

$$
\left(b_{1}(t), \cdots, b_{k}(t)\right)^{T}=V \Lambda^{t} V^{-1} e_{k} .
$$

In (4.2), $\Lambda^{t}$ is defined to be the diagonal $k \times k$ matrix whose entries on the main diagonal are $\pi_{1}^{t}, \pi_{2}^{t}, \cdots, \pi_{k}^{t}$. It now follows that, since $e_{1}, \cdots, e_{s}$ is a basis in $E^{s}(s \leqq n)$, if

$$
p=\alpha_{1} x+\alpha_{2} x^{2}+\cdots+\alpha_{s} x^{s},
$$

then

$$
B_{n}^{(t)}(p)=\sum_{i=1}^{s} \alpha_{i} B_{n}^{(t)}\left(x^{i}\right)
$$

Moreover, if we define

$$
B_{n}^{(t)}(c)=c
$$

and

$$
B_{n}^{(t)}(c+p)=c+B_{n}^{(t)}(p)
$$

where $c$ is a constant and $p$ is given by (4.3), then we obtain

$$
B_{n}^{(t)}(p)=\sum_{i=0}^{s} \alpha_{i} B_{n}^{(t)}\left(x^{i}\right)
$$

when

$$
p=\alpha_{0}+\alpha_{1} x+\cdots+\alpha_{s} x^{s} .
$$

We observe further that if $-\infty<u<\infty$, then

$$
\Lambda^{u+t}=\Lambda^{u} \Lambda^{t}
$$

and so it is easy to see that

$$
B_{n}^{(t+u)}\left(x^{k}\right)=B_{n}^{(t)}\left(B_{n}^{(u)}\left(x^{k}\right)\right)=B_{n}^{(u)}\left(B_{n}^{(t)}\left(x^{k}\right)\right),
$$

and hence

$$
B_{n}^{(t+u)}(p)=B_{n}^{(t)}\left(B_{n}^{(u)}(p)\right)=B_{n}^{(u)}\left(B_{n}^{(t)}(p)\right)
$$

for any polynomial $p$ of degree at most $n$.

If $f$ is bounded on $[0,1]$, we can now define

$$
B_{n}^{(t)}(f)=B_{n}^{t-1}\left(B_{n}(f)\right) .
$$

This definition focuses attention on the case $t=0$. The polynomial 
of degree at most $n$

$$
B_{n}^{*}(f)=B_{n}^{(0)}(f)=B_{n}^{-1}\left(B_{n} f\right)
$$

is a kind of surrogate $f$. How is this polynomial related to $f$ ? It is clear that if $f=p$, a polynomial of degree at most $n$, then

$$
B_{n}^{*} p=p \text {. }
$$

In particular, let $p=L_{n}(f)$ be the unique polynomial of degree at most $n$ which agrees with $f(x)$ at $x=j / n, j=0, \cdots, n$. Then $B_{n}(f)=$ $B_{n}\left(L_{n}(f)\right)$ and so

$$
B_{n}^{*}(f)=B_{n}^{*}\left(L_{n}(f)\right)=L_{n}(f) .
$$

Of course, this result could have been obtained without the apparatus of this paper, but it comes out of our discussion quite naturally.

We wish to thank Benjamin Weiss for some helpful advice on this work.

\section{REFERENCES}

1. C. Jordan, Calculus of Finite Differences, 2nd ed., New York, 1950.

2. G. G. Lorentz, Bernstein Polynomials, Toronto, 1953.

3. I. J. Schoenberg, On Variation Diminishing Approximation Methods, On Numerical Approximation, R. E. Langer, ed., Madison, 1959.

Received October 6, 1965.

IBM Watson Research Center

YORKTOWN HEIGHTS, NEW YORK 


\section{PACIFIC JOURNAL OF MATHEMATICS}

\section{EDITORS}

\section{H. SAMELSON}

Stanford University

Stanford, California

\author{
J. P. JANS \\ University of Washington \\ Seattle, Washington 98105
}

\section{J. DuGUNDJI}

University of Southern California

Los Angeles, California 90007

RichaRd ARENS

University of California

Los Angeles, California 90024

\section{ASSOCIATE EDITORS}

E. F. BECKENBACH
B. H. NeUManN

F. WOLF

K. YOSIDA

\section{SUPPORTING INSTITUTIONS}

UNIVERSITY OF BRITISH COLUMBIA

CALIFORNIA INSTITUTE OF TECHNOLOGY

UNIVERSITY OF CALIFORNIA

MONTANA STATE UNIVERSITY

UNIVERSITY OF NEVADA

NEW MEXICO STATE UNIVERSITY

OREGON STATE UNIVERSITY

UNIVERSITY OF OREGON

OSAKA UNIVERSITY

UNIVERSITY OF SOUTHERN CALIFORNIA
STANFORD UNIVERSITY

UNIVERSITY OF TOKYO

UNIVERSITY OF UTAH

WASHINGTON STATE UNIVERSITY

UNIVERSITY OF WASHINGTON

AMERICAN MATHEMATICAL SOCIETY

CHEVRON RESEARCH CORPORATION

TRW SYSTEMS

NAVAL ORDNANCE TEST STATION

Mathematical papers intended for publication in the Pacific Journal of Mathematics should be typewritten (double spaced). The first paragraph or two must be capable of being used separately as a synopsis of the entire paper. It should not contain references to the bibliography. Manuscripts may be sent to any one of the four editors. All other communications to the editors should be addressed to the managing editor, Richard Arens at the University of California, Los Angeles, California 90024.

50 reprints per author of each article are furnished free of charge; additional copies may be obtained at cost in multiples of 50 .

The Pacific Journal of Mathematics is published monthly. Effective with Volume 16 the price per volume ( 3 numbers) is $\$ 8.00$; single issues, $\$ 3.00$. Special price for current issues to individual faculty members of supporting institutions and to individual members of the American Mathematical Society: $\$ 4.00$ per volume; single issues $\$ 1.50$. Back numbers are available.

Subscriptions, orders for back numbers, and changes of address should be sent to Pacific Journal of Mathematics, 103 Highland Boulevard, Berkeley 8, California.

Printed at Kokusai Bunken Insatsusha (International Academic Printing Co., Ltd.), No. 6, 2-chome, Fujimi-cho, Chiyoda-ku, Tokyo, Japan.

PUBLISHED BY PACIFIC JOURNAL OF MATHEMATICS, A NON-PROFIT CORPORATION

The Supporting Institutions listed above contribute to the cost of publication of this Journal, but they are not owners or publishers and have no responsibility for its content or policies. 


\section{Pacific Journal of Mathematics}

\section{Vol. 21, No. $3 \quad$ BadMonth, 1967}

Richard Allen Askey, A transplantation theorem for Jacobi coefficients . . . 393

Raymond Balbes, Projective and injective distributive lattices .......... 405

Raymond Balbes and Alfred Horn, Order sums of distributive lattices . . . . 421

Donald Charles Benson, Nonconstant locally recurrent functions ........ 437

Allen Richard Bernstein, Invariant subspaces of polynomially compact operators on Banach space ............................... 445

Robert F. Brown, Fixed points and fibre ................... 465

David Geoffrey Cantor, On the Stone-Weierstrass approximation theorem for valued fields ................................ 473

James Walton England, Stability in topological dynamics .............. 479

Alessandro Figà-Talamanca and Daniel Rider, A theorem on random

Fourier series on noncommutative groups.................. 487

Sav Roman Harasymiv, A note of dilations in $L^{p} \ldots \ldots \ldots \ldots \ldots \ldots . \ldots 493$

J. G. Kalbfleisch, A uniqueness theorem for edge-chromatic graphs ....... 503

Richard Paul Kelisky and Theodore Joseph Rivlin, Iterates of Bernstein polynomials .................................... 511

D. G. Larman, On the union of two starshaped sets ............... 521

Henry B. Mann, Josephine Mitchell and Lowell Schoenfeld, Properties of differential forms in $n$ real variables ...................... 525

John W. Moon and Leo Moser, Generating oriented graphs by means of team comparisons .

Veikko Nevanlinna, A refinement of Selberg's asymptotic equation ...

Ulrich Oberst, Relative satellites and derived functors of functors with additive domain ..............................

John Vincent Ryff, On Muirhead's theorem...............

Carroll O. Wilde and Klaus G. Witz, Invariant means and the Stone-Čech compactification 